\title{
Effects of erythropoietin and methylprednisolone on AQP4 expression in astrocytes
}

\author{
CHANGCHAO WANG ${ }^{1}$, YOUJA XU ${ }^{1}$, YADONG HUANG $^{2}$ and YAN HUANG ${ }^{2}$ \\ ${ }^{1}$ Department of Orthopedics, The Second Hospital Affiliated to Soochow University, Suzhou, Jiangsu 215004; \\ ${ }^{2}$ Jiangsu Food and Pharmaceutical Science College, Huai'an, Jiangsu 223003, P.R. China
}

Received August 12, 2016; Accepted May 18, 2017

DOI: $10.3892 / \mathrm{mmr} .2017 .7330$

\begin{abstract}
Methylprednisolone sodium succinate (MPSS) has been suggested as a treatment for spinal cord injury (SCI), but its use has been limited due to its adverse effects. Erythropoietin (EPO) has been suggested as a promising candidate for limiting SCI in mammals. The aim of the present study was to investigate the effects of EPO in combination with MPSS on astrocytes following ischemic injury in vitro. Astrocytes were isolated from the cerebral cortex of postnatal day 3 Sprague-Dawley rats and cultured in vitro. Astrocyte ischemic injury was induced by oxygen and glucose deprivation for $4 \mathrm{~h}$, and reperfusion was simulated by subsequent culture under normoxic conditions. The effects of EPO and MPSS on the expression of aquaporin-4 (AQP4) were investigated. Ischemic astrocytes were treated with EPO $(10 \mathrm{U} / \mathrm{ml})$, MPSS $(10 \mu \mathrm{g} / \mathrm{ml})$, or EPO $(10 \mathrm{U} / \mathrm{ml})$ in combination with MPSS $(10 \mu \mathrm{g} / \mathrm{ml})$ during reperfusion. The cell viability of astrocytes was assessed using an MTT assay. The mRNA and protein expression levels of AQP4 were determined using reverse transcription-quantitative polymerase chain reaction and western blot analysis, respectively. The role of the protein kinase $\mathrm{C}$ (PKC) signaling pathway in the molecular mechanisms underlying the effects of EPO and MPSS was also investigated. The present results demonstrated that following treatment with EPO and MPSS, the mRNA expression levels of AQP4 were upregulated and cell viability was enhanced. EPO and MPSS effectively inhibited the oxygen and glucose deprivation-mediated downregulation of AQP4 following reperfusion. In addition, the combined treatment with EPO and MPSS exhibited higher AQP4 expression levels and cell viability compared with each treatment alone. Finally, the effects of EPO and MPSS on AQP4 expression were partially reversed by pretreatment with the PKC inhibitor Ro 31-8220.
\end{abstract}

Correspondence to: Dr Changchao Wang, Department of Orthopedics, The Second Hospital Affiliated to Soochow University, 1055 Sanxiang Road, Suzhou, Jiangsu 215004, P.R. China

E-mail: wangchangchao@126.com

Key words: erythropoietin, astrocytes, ischemia-reperfusion injury, methylprednisolone, aquaporin-4
The present study indicated that EPO and MPSS had a synergistic effect on AQP4 expression following reperfusion, and suggest that they may be combined in the treatment of SCI.

\section{Introduction}

Central nervous system (CNS) ischemic injury is a major cause of morbidity and mortality. Reperfusion injury is caused by the recovery of blood supply to the tissues following a period of ischemia. The shortage of blood supply creates a condition of nutrient and oxygen insufficiency, and the restoration of blood circulation results in oxidative damage in the tissues (1). Astrocytes are glial cells in the CNS and serve important roles in numerous CNS functions, including neuronal regeneration, apoptosis regulation, blood-brain barrier (BBB) maintenance and extracellular ion homeostasis (2).

Edema is among the most prominent features in spinal cord injury (SCI) and has been attributed to astrocyte dysfunction (3). Under conditions of cellular edema, astrocytes have been reported to fail to exert their physiological functions to support neuronal activity (4). Following SCI in humans, the recovery process has been demonstrated to involve the rapid transition of astrocytes from an aquaporin-4 (AQP4)-positive to an AQP4-negative state, which has been associated with the development of spinal cord edema (5). AQP4 is expressed by astrocytic end-processes that encircle microvessels along the $\mathrm{BBB}$ and the brain-cerebrospinal fluid interface (6). AQP4 is a water channel that is functionally enriched on astrocytic end-processes at the perivascular space and the subpial surface of the glia limitans (7). AQP4 selectively conducts water molecules in and out of astrocytes during edema formation and resolution, while preventing the passage of ions and other solutes (8). Enhanced AQP4 expression has been associated with increased CNS water contents and the development of edema following ischemia (9). However, contradictory reports have demonstrated that AQP4 mitigates the damage and suppresses the retrograde degeneration of rubrospinal neurons, via promoting the resolution of edema following SCI (10). AQP4 has also been reported to facilitate the clearance of excess water following contusion-induced SCI (11). The regulation of AQP4 expression is critical for tissue water transport during repair processes in the CNS following ischemic injury, in the maintenance of the $\mathrm{BBB}$ and in the release and uptake of neurotransmitters (12). 
Methylprednisolone sodium succinate (MPSS) has been used as a treatment for acute SCI (13); however, high doses of MPSS have been associated with deleterious adverse effects (14), such as enhancing the avascular necrosis of nerve fibers (15). The use of MPSS in the clinical treatment of acute SCI was prompted by its protective effects on neurological damage (16). However, high doses of MPSS have been associated with adverse effects, including infections, sepsis, respiratory complications and an increase in mortality (17). Reduction of the administered MPSS dose, or its substitution with alternative neuroprotective agents, may have potential as strategies to avoid the adverse effects of MPSS during the treatment of SCI (18).

Ischemia-reperfusion (IR) injury causes edema that further limits the blood supply to the tissues and results in hypoxia (19). Under hypoxic conditions, the synthesis of erythropoietin (EPO) by interstitial cells of the renal cortex is potentiated. EPO has been reported to exert neuroprotective effects against SCI (20); however, EPO has been associated with adverse effects, such as increases in hematocrit and blood viscosity, and thrombosis. EPO has been identified as a novel neuroprotective agent against neurological injury (21). EPO and its receptor (EPO-R) are expressed throughout the CNS, including astrocytes, and have been reported to exert neuroprotection in cell lines and animal models of CNS disorders (22). Previous studies demonstrated that intravenous administration of EPO within $8 \mathrm{~h}$ following non-hemorrhagic cerebral infarction significantly improved prognosis in rats (23). Notably, Gorio et al (24) suggested that MPSS combined with EPO may suppress the protective effects of EPO against neuronal injury, via blocking the EPO-R, increasing vascular EPO clearance and inhibiting the EPO-mediated synthesis of neurotrophic factors. However, contradictory reports demonstrated that combination treatment with EPO and MPSS following nerve injury potentiated the recovery of neurological function and improved tissue pathology (25). The present study aimed to investigate the effects of the combination of EPO with MPSS in the treatment of SCI, via assessing their effects on AQP4 expression in astrocytes following ischemia and reperfusion in vitro.

\section{Materials and methods}

Primary astrocyte culture. The present study obtained ethical approval by the Soochow University Ethics Committee (Suzhou, China). Newborn female Sprague-Dawley rats (age, 1-3 days) were purchased from the Laboratory Animal Center of Soochow University and housed under controlled conditions of temperature $\left(22^{\circ} \mathrm{C}\right)$, humidity, and light (light/dark cycle, 14/10 h). Rats were exclusively breastfed until treatment. Postnatal day (P) 3 female Sprague-Dawley rats were sacrificed by decapitation. The hypothalamus was isolated, minced and washed with PBS to remove the meninges and residual blood, and then digested and dissociated using $0.25 \%$ trypsin for $3 \mathrm{~min}$ (Gibco; Thermo Fisher Scientific, Inc., Waltham, MA, USA) in a $37^{\circ} \mathrm{C}$ water bath. Dissociated cells were filtered through a $30-\mu \mathrm{m}$ nylon mesh, and then seeded onto poly-L-lysine $(10 \mu \mathrm{g} / \mathrm{ml}$; Sigma-Aldrich; Merck KGaA, Darmstadt, Germany) pretreated $75-\mathrm{cm}^{2}$ culture dishes. Cells were cultured in Dulbecco's modified Eagle's medium/Nutrient
Mixture F-12 (DMEM/F12; Gibco; Thermo Fisher Scientific, Inc.) supplemented with $10 \%$ fetal bovine serum (FBS; Hangzhou Sijiqing Biological Engineering Materials Co., Ltd.) and maintained at $37^{\circ} \mathrm{C}$ in a $5 \% \mathrm{CO}_{2}$ incubator. The culture medium was replaced every 2-3 days, until the cells achieved the desired confluence following $\sim 2$ weeks in culture, as previously described (26). Microglial cells and oligodendrocyte progenitors were dissociated following agitation in a $37^{\circ} \mathrm{C}$ incubator at $260 \mathrm{rpm}$ for $2 \mathrm{~h}$ and at $200 \mathrm{rpm}$ overnight. The microglial cells and oligodendrocytes grow on the surface of the astrocytes and were easier to detach by agitation; the astrocytes remained attached to the plate. Cells of the 3rd passage were prepared for testing and cells were purified after 2-3 passages.

Characterization of astrocytes. Astrocytes were identified by immunocytochemical examination using anti-glial fibrillary acidic protein (GFAP) antibody, which revealed obvious astrocytic morphology. Cell nuclei were counterstained with DAPI. Astrocyte purity was assessed based on the $\%$ of GFAP-positive cells to DAPI-positive cells and was revealed to be $>95 \%$.

Briefly, cells were seeded $\left(1-5 \times 10^{6}\right.$ cells $\left./ \mathrm{ml}\right)$ into 6-well plates, fixed with $4 \%$ paraformaldehyde at $22^{\circ} \mathrm{C}$ for $20 \mathrm{~min}$, permeabilized with $0.01 \%$ Triton X-100 and blocked in 5\% goat serum (G9023, Sigma-Aldrich; Merck KGaA) for $2 \mathrm{~h}$ at $4^{\circ} \mathrm{C}$. Cells were then incubated with rat anti-GFAP primary antibody overnight at $4^{\circ} \mathrm{C}(1: 1,000$; sc-9065; Santa Cruz Biotechnology, Inc., Dallas, TX, USA), and with sheep anti-rat Cyanine 3-conjugated secondary antibody [1:100; D111042; Sangon Biotech (Shanghai) Co., Ltd., Shanghai, China] at $24^{\circ} \mathrm{C}$ for $1 \mathrm{~h}$. Cells were counterstained with the fluorescent dye DAPI for 15 min, and then dehydrated by graded concentrations of ethanol. Stained cells were observed under an epifluorescence microscope (Olympus Corporation, Tokyo, Japan) and photomicrographs were captured. Three randomly selected fields of view/slide were used for cell counting by Image Pro Plus 6.0 (National Institutes of Health, Bethesda, MD, USA).

IR injury. IR injury includes two phases: An acute phase of ischemic injury, and a delayed phase of reperfusion injury. To simulate ischemic conditions, primary astrocyte cultures were transiently deprived of oxygen and glucose. The medium was replaced with oxygen-depleted, glucose-, serum- and phenol red-free DMEM (Gibco; Thermo Fisher Scientific, Inc.) and the cells were incubated in a $92 \% \mathrm{~N}_{2}, 5 \% \mathrm{CO}_{2}$ and $1 \%$ $\mathrm{O}_{2}$ incubator at $37^{\circ} \mathrm{C}$. Following $4 \mathrm{~h}$ of oxygen and glucose deprivation, reperfusion was simulated. The medium was restored to DMEM/F12 supplemented with $10 \%$ FBS and cells were cultured in a normoxic incubator $\left(5 \% \mathrm{CO}_{2}\right)$ at $37^{\circ} \mathrm{C}$, as previously described (27). Normal cells were not subjected to oxygen and glucose deprivation and were maintained in a normoxic incubator at $37^{\circ} \mathrm{C}$.

Experimental groups. The following treatment groups were used in the present study: Normal group, untreated astrocytes maintained in normoxic conditions; Control group, cultured astrocytes that were subjected to $4 \mathrm{~h}$ of oxygen and glucose deprivation, and subsequently cultured in regular medium 
under normoxic conditions; EPO group, cultured astrocytes that were subjected to $4 \mathrm{~h}$ of oxygen and glucose deprivation, followed by reperfusion in the presence of $10 \mathrm{U} / \mathrm{ml} \mathrm{EPO}$ (Kirin brewery Company, Ltd., Tokyo, Japan); MPSS group, cultured astrocytes that were subjected to $4 \mathrm{~h}$ of oxygen and glucose deprivation, followed by reperfusion in the presence of $10 \mu \mathrm{g} / \mathrm{ml}$ MPSS (Pfizer Inc., New York, NY, USA); EPO + MPSS group, cultured astrocytes that were subjected to $4 \mathrm{~h}$ of oxygen and glucose deprivation, followed by reperfusion in the presence of $10 \mathrm{U} / \mathrm{ml}$ EPO and $10 \mu \mathrm{g} / \mathrm{ml}$ MPSS.

MTT assay. The MTT assay was used to determine the viability of astrocytes according to the measured absorbance or optical density (OD) value. Cells were seeded $\left(5 \times 10^{4}\right.$ cells $\left./ \mathrm{ml}\right)$ into 96-well plates in culture medium (200 $\mu \mathrm{l} /$ well). A total of $20 \mu 1$ MTT solution was added to each well and cells were incubated for an additional $4 \mathrm{~h}$. Subsequently, $150 \mu \mathrm{l}$ dimethyl sulfoxide were added to each well and plates were agitated gently for $10 \mathrm{~min}$ to fully solubilize the formazan crystals. The absorbance of each well was measured at $490 \mathrm{~nm}$.

Reverse transcription-quantitative polymerase chain reaction (RT-qPCR). Total RNA was extracted from cells using TRIzol reagent (Invitrogen; Thermo Fisher Scientific, Inc.). The concentration and purity of RNA was evaluated by spectrometry at 260 and $280 \mathrm{~nm}$. Total RNA was reverse transcribed into cDNA using a SYBR Green Real-Time PCR Master Mix (Thermo Fisher Scientific, Inc.) according to the manufacturer's protocol. The amplification conditions were as follows: AQP4, $45 \mathrm{sec}$ at $94^{\circ} \mathrm{C}$ followed by 30 cycles of $30 \mathrm{sec}$ at $94^{\circ} \mathrm{C}, 30 \mathrm{sec}$ at $52^{\circ} \mathrm{C}$ and $60 \mathrm{sec}$ at $72^{\circ} \mathrm{C} ; \beta$-actin, $45 \mathrm{sec}$ at $94^{\circ} \mathrm{C}$ followed by 30 cycles of $30 \mathrm{sec}$ at $94^{\circ} \mathrm{C}, 30 \mathrm{sec}$ at $58^{\circ} \mathrm{C}$ and $60 \mathrm{sec}$ at $72^{\circ} \mathrm{C}$. Primers sequences, designed by Primer-Express V3.0 (Thermo Fisher Scientific, Inc.), were as follows: AQP4 forward, 5'-TTGGACCAATCATAGGCGC-3' and reverse, 5'-GGTCAATGTCGATCACATGC-3' (product, $213 \mathrm{bp}) ; \beta$-actin forward, 5'-GAGAGGGAAATCGTGCGT GAC-3' and reverse, 5'-CATCTGCTGGAAGGTGGACA-3' (product, $457 \mathrm{bp}$ ). The experiments were performed in triplicate and the comparative Cq method (28) was used to perform relative quantification. Differential expression was calculated by analysis of the target gene amplification following normalization to the rat $\beta$-actin endogenous reference.

Western blot analysis. Cells from the various treatment groups were collected and lysed using Western/IP Cell Lysis Buffer (Beyotime Institute of Biotechnology, Haimen, China) for $1 \mathrm{~h}$ at $0^{\circ} \mathrm{C}$. Protein concentration was measured by bicinchoninic acid assay. Equal amounts of extracted protein samples $(50 \mu \mathrm{g})$ were separated by $10 \%$ SDS-PAGE and transferred onto polyvinylidene difluoride membranes. The membranes were blocked with $2 \%$ skimmed milk and TBS-0.1\% Tween-20 (TBST). Primary polyclonal antibodies against AQP4 (sc-9888; Santa Cruz Biotechnology, Inc., Dallas, TX, USA) diluted (1:500) in TBST were used. Membranes were incubated with the primary antibodies at $4^{\circ} \mathrm{C}$ overnight, or with anti-GAPDH [1:1,000; AB10016; Sangon Biotech (Shanghai) Co., Ltd] at $4^{\circ} \mathrm{C}$ for $2 \mathrm{~h}$. Blots were subsequently incubated with horseradish peroxidase-conjugated rabbit anti-goat IgG secondary antibodies [1:1,000; D111047; Sangon (Shanghai) Biotech Co.,
Ltd.] for $2 \mathrm{~h}$ at $4^{\circ} \mathrm{C}$. Protein bands were visualized on X-ray film using enhanced chemiluminescence (luminol chemiluminescence substrate, SF-2; Jiangsu Sunlant Bioengineering Co., Ltd.). Developed films were digitized and semi-quantified by densitometry using a gel imaging system (Smartview-2001 Version 5; Shanghai Furi Science \& Technology Co., Ltd., Shanghai, China).

Statistical analysis. Data are expressed the mean \pm standard deviation. Statistical analysis was performed with one-way analysis of variance and the Student-Newman-Keuls post hoc test were performed using SPSS software version 16.0 (SPSS, Inc., Chicago, IL, USA). $\mathrm{P}<0.05$ was considered to indicate a statistically significant difference.

\section{Results}

Astrocyte characterization. Astrocytes of the third passage were observed by microscopy. Astrocytes exhibited 'vascular processes', which connected them to the outer surface of the capillary walls in close proximity. Astrocytic processes were revealed to envelope neuronal synapses. Individual synapses were viewed as slender structures that were interwoven into a mesh. Astrocytes were demonstrated to extend processes in order to form the glial limiting membrane. Following immunostaining for GFAP, an astrocyte marker, cells exhibited a characteristic astrocytic morphology and GFAP-positive staining (Fig. 1). DAPI was used to counterstain the nuclei of living cells (Fig. 1). The purity of the cultures was calculated as the $\%$ of GFAP-positive cells over the total number of cells (based on DAPI staining). Astrocytes were revealed to amount for $\sim 95 \%$ of the culture.

Astrocyte viability. The cell viability of astrocytes in the five experimental groups tested in the present study was evaluated by MTT assay. The total numbers of viable cells were significantly increased in all the drug intervention groups (Fig. 2). The results demonstrated that treatment with EPO, MPSS or their combination significantly promoted viability of astrocytes compared with the control (Fig. 2). Of note, the cell viability of the EPO + MPSS group, was significantly increased compared with EPO or MPSS groups alone (Fig. 2).

AQP4 mRNA expression levels. To analyze the changes of AQP4 gene expression as a function of time, RT-qPCR analysis was performed. A time effect curve was constructed from results up to $18 \mathrm{~h}$ following reperfusion (Fig. 3). AQP4 mRNA levels had the most obvious changes at $0.5 \mathrm{~h}$ following reperfusion, and therefore this time point was chosen for RT-qPCR analysis of all the five experimental groups (Fig. 4). Notably, AQP4 expression following EPO or MPSS intervention was significantly upregulated at $0.5 \mathrm{~h}$, compared with the control group (Fig. 4). In addition, the EPO + MPSS group had significantly higher AQP4 mRNA expression levels compared with the control group, and compared with either intervention alone (Fig. 4), indicating a synergistic action of the two drugs in upregulating AQP4 expression.

AQP4 protein expression levels. Western blot analysis was used in order to examine the time effect curve of AQP4 


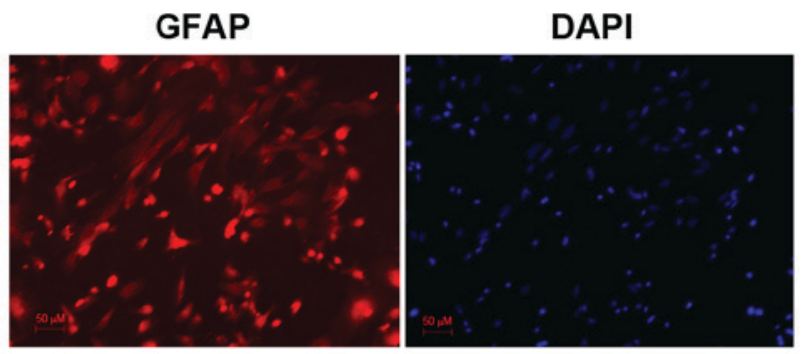

Figure 1. Astrocyte characterization. Cell cultures were immunostained for GFAP (red) and counterstained with DAPI (blue). GFAP-positive astrocytes were revealed to amount for $\sim 95 \%$ of total cells. Scale bar, $50 \mu \mathrm{m}$. GFAP, glial fibrillary acidic protein.

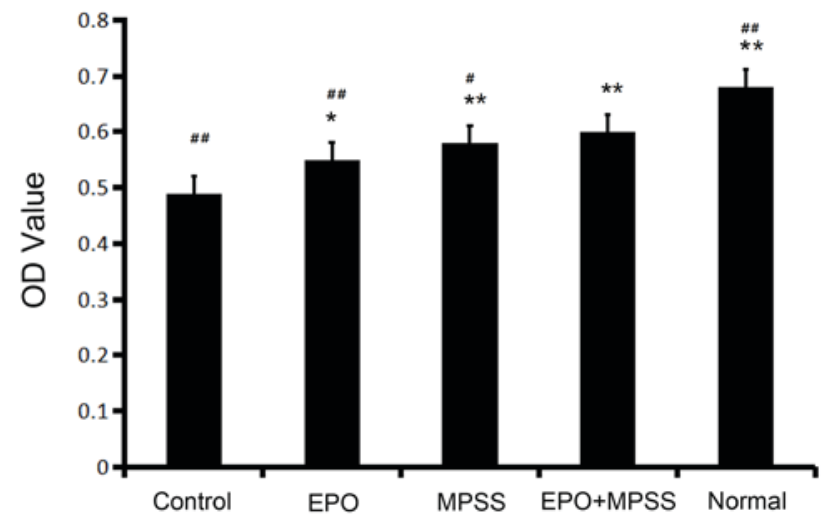

Figure 2. Effects of EPO and MPSS treatment on astrocyte cell viability following reperfusion. Numbers of viable cells were measured by MTT assay following reperfusion for $4 \mathrm{~h}(\mathrm{n}=8)$. ${ }^{*} \mathrm{P}<0.05$ and ${ }^{* *} \mathrm{P}<0.01$ vs. control group; ${ }^{\#} \mathrm{P}<0.05$ and ${ }^{\# \#} \mathrm{P}<0.01$ vs. MPSS+EPO group. EPO, erythropoietin; MPSS, methylprednisolone sodium succinate; OD, optical density.

protein expression levels for $15 \mathrm{~h}$ following reperfusion. Levels of AQP4 protein were upregulated beginning at $12 \mathrm{~h}$ following reperfusion with drug interventions (Fig. 5). Thus, the time of $12 \mathrm{~h}$ after drug intervention was selected for further analysis of the five experimental groups (Fig. 6). Compared with the normal group, astrocyte AQP4 protein expression levels in the control group decreased significantly following oxygen and glucose deprivation (Fig. 6). Following reperfusion, AQP4 protein levels increased significantly by treatment with EPO, MPSS or their combination, compared with control (Fig. 6). Notably, the AQP4 protein levels were significantly higher in the EPO + MPSS group compared with either drug alone (Fig. 6), confirming a synergistic action between EPO and MPSS in the regulation of AQP4 expression.

Ro 31-8220 inhibits the EPO and MPSS-mediated AQP4 upregulation. Cultured astrocytes were subjected to oxygen and glucose deprivation for $4 \mathrm{~h}$, and then pretreated with the protein kinase C (PKC) inhibitor Ro 31-8220 (100 nM, Medchemexpress) for $10 \mathrm{~min}$ followed by reperfusion. Western blot analysis demonstrated that the protein expression levels of AQP4 were significantly decreased in the control compared with the normal group $(\mathrm{P}<0.05$; Fig. 6$)$, and PKC inhibition had no effect in the control group. However, in the EPO and the MPSS groups, Ro 31-8220 pretreatment significantly

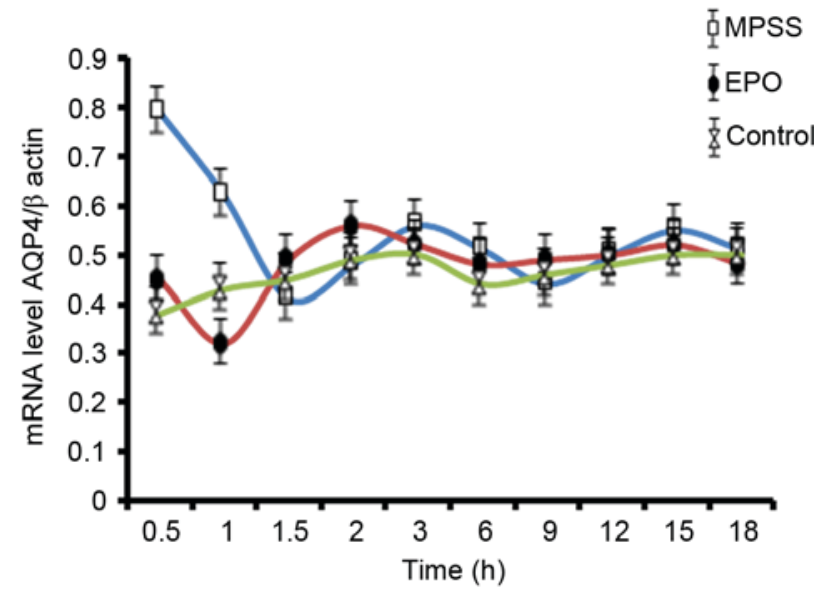

Figure 3. AQP4 mRNA expression levels were measured at the indicated time points following reperfusion using reverse transcription-quantitative polymerase chain reaction $(n=3)$. AQP4, aquaporin 4; EPO, erythropoietin; MPSS, methylprednisolone sodium succinate.

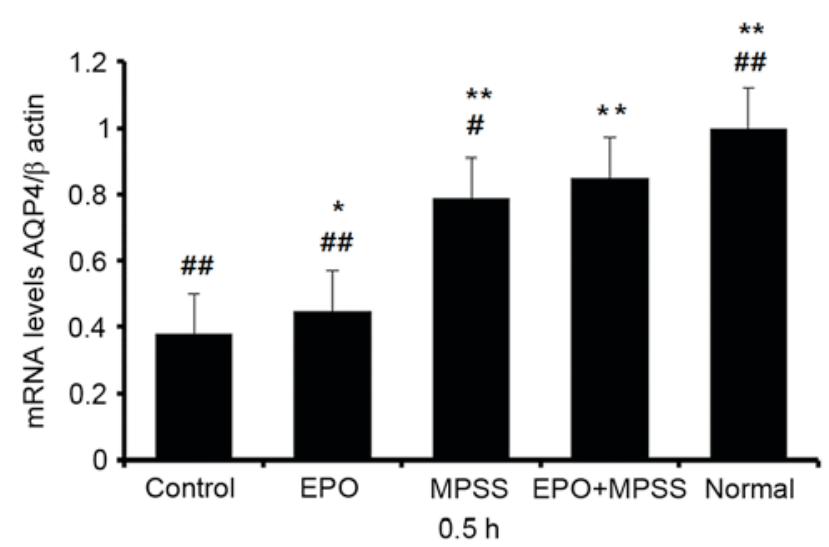

Figure 4. Effects of EPO and MPSS on AQP4 mRNA expression levels following reperfusion. AQP4 mRNA expression levels were measured in all experimental groups at $0.5 \mathrm{~h}$ following reperfusion using reverse transcription polymerase chain reaction $(n=9)$. ${ }^{*} \mathrm{P}<0.05$ and ${ }^{* *} \mathrm{P}<0.01$ vs. control group. ${ }^{\#} \mathrm{P}<0.05$ and ${ }^{\# \#} \mathrm{P}<0.01$ vs. MPSS+EPO group. EPO, erythropoietin; MPSS, methylprednisolone sodium succinate; AQP4, aquaporin 4.

decreased the AQP4 protein expression levels, compared with cells treated with EPO or MPSS alone ( $\mathrm{P}<0.01$; Fig. 6). Finally, the EPO + MPSS group exhibited the highest protein expression levels for AQP4 following reperfusion, and PKC inhibition partially but significantly inhibited this AQP4 upregulation $(\mathrm{P}<0.05$; Fig. 6).

\section{Discussion}

In the present study, primary astrocyte cultures were established following the isolation of astrocytes from the rat cerebral cortex; $\mathrm{P} 3$ rats were used, as the yield of viable neurons in cell suspension is the highest at this stage. Astrocytes isolated from female rats have been reported to be more resistant to oxygen and glucose deprivation compared with male astrocytes (29). In the present study, astrocytes were obtained from P3 female rats and were subjected to oxygen and glucose deprivation followed by reperfusion, in accordance with a widely-used model to imitate ischemic conditions in vitro (30). 

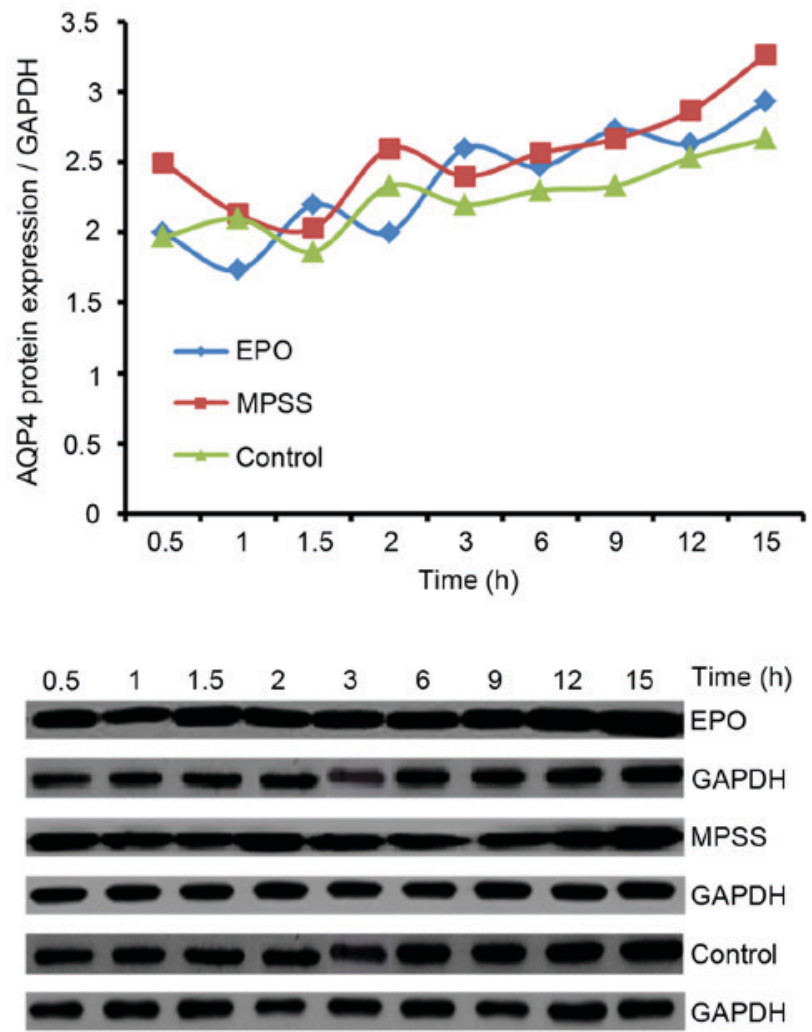

Figure 5. AQP4 protein expression levels were measured at the indicated time points following reperfusion using western blot analysis $(n=3)$. AQP4, aquaporin 4; EPO, erythropoietin; MPSS, methylprednisolone sodium succinate.

In the present study, EPO and MPSS in combination attenuated the ischemia-induced downregulation of AQP4 expression, and were revealed to be more effective compared with the administration of EPO or MPSS therapy alone. The mRNA expression levels of AQP4 were significantly downregulated in astrocytes following ischemia, thus suggesting that the decreased expression of AQP4 may limit water influx so as to maintain the physiological morphology and preserve the viability of astrocytes under ischemic conditions.

In the early stages of cytotoxic edema, AQP4 expression is rapidly increased, leading to water influx following reperfusion, and the development of cytotoxic edema. The upregulation in AQP4 expression causes excess water influx into the cells, thus resulting in cell swelling and elevated pressure (31). In the present study, the results demonstrated that in the initial $30 \mathrm{~min}$ of reperfusion, the oxygen and glucose deprivation-mediated downregulation in AQP4 expression was inhibited by EPO or MPSS administration. In addition, the mRNA and protein expression levels of AQP4 in the EPO + MPSS group were significantly higher compared with the control group. The present results were in accordance with previous animal studies using experimental models of SCI, which reported that when EPO was administered 30 min post-injury, it could improve the neurological outcome (32).

The upregulation of AQP4 expression has been observed in hypoxic-ischemic neonatal rats following treatment with EPO (33). Conversely, EPO was demonstrated to significantly attenuate the upregulation in AQP4 expression induced by oxygen-glucose deprivation followed by reoxygenation (34). The protective effects of EPO in astrocytes may be mediated
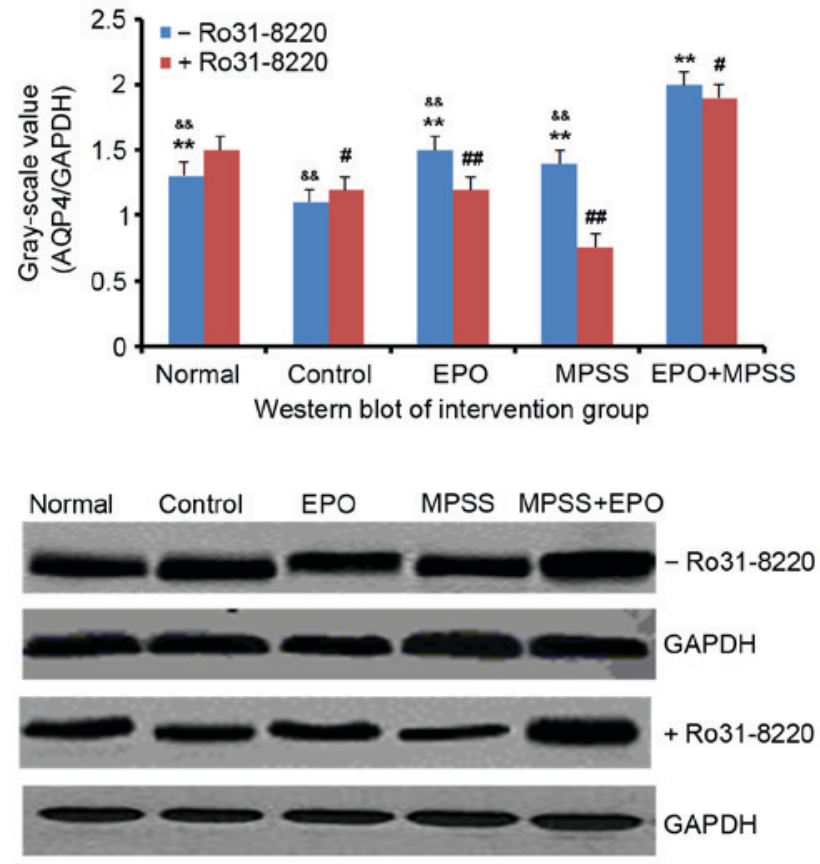

Figure 6. Effects of PKC inhibition on EPO and MPSS-induced AQP4 protein expression following reperfusion. Western blot analysis was used to assess the protein expression levels of AQP4 in astrocytes following $12 \mathrm{~h}$ reperfusion with the indicated drug interventions $(n=3)$. The PKC inhibitor Ro 31-8220 was used to investigate the implication of PKC-mediated signaling pathways. ${ }^{\#} \mathrm{P}<0.05$ and ${ }^{\# \#} \mathrm{P}<0.01+$ Ro vs. $-\mathrm{Ro} ;{ }^{* *} \mathrm{P}<0.01$ vs. control $-\mathrm{Ro}$; ${ }^{\&} \mathrm{P}<0.01$ vs. EPO+MPSS -Ro. PKC, protein kinase C; EPO, erythropoietin; MPSS, methylprednisolone sodium succinate; AQP4, aquaporin 4 .

through the activation of c-Jun N-terminal kinase, mitogen-activated protein kinase and phosphatidylinositol-4,5-bisphosphate 3-kinase/Akt signaling pathways $(35,36)$. EPO treatment has been demonstrated to preserve the neurological function and to attenuate neuronal cell damage in a mouse model of spinal cord IR injury (37), and it has been reported to significantly suppress edema formation and prevent neurological injury following spinal cord ischemia in rats (38). Furthermore, EPO administration has been reported to protect the integrity of the $\mathrm{BBB}$, and its beneficial effects during BBB disruption have been associated with increased levels of AQP4 (39-41).

In the present study, the mRNA expression levels of AQP4 were significantly increased following treatment with MPSS compared with the control group. EPO inhibited the rapid increase in AQP4 expression more potently compared with MPSS. By contrast, MPSS inhibited the rapid decrease more effectively than did EPO. Previous studies have reported that the inhibition of the initial upregulation of AQP4 prevents the development of cellular edema (42). The AQP4 mRNA level is biphasic following reperfusion. Triggered by SCI, an early upregulation of AQP4 expression has been reported, suggested to facilitate edema formation, followed by a later downregulation after the onset of edema (43). The present study demonstrated that EPO and MPSS displayed protection within $30 \mathrm{~min}$.

$\mathrm{PKC}$ has been reported to regulate AQP4 protein expression, and PKC activation by EPO can alter plasma membrane water permeability (34). Low doses of MPSS have been demonstrated to elicit a biological response through the activation of the same signaling pathway (44). The activation of PKC causes a decrease in AQP4 expression, thus reducing cellular water 
permeability and alleviating ischemia-induced swelling (34). In the present study, in order to investigate the roles of PKC in the mechanisms underlying the effects of EPO and MPSS on IR injury, astrocytes were pretreated with the PKC inhibitor Ro 31-8220 prior to reperfusion. The present results revealed that PKC inhibition partly counteracted the effects of EPO on AQP4 expression following ischemia. Notably, Ro 31-8220 significantly inhibited the MPSS-induced AQP4 upregulation, thus suggesting that EPO and MPSS may act through a common mechanism implicating the PKC pathway. However, it is possible that other pathways may also be implicated in the regulation of osmotic pressure in astrocytes, and further studies are required to fully elucidate the molecular mechanisms of EPO and MPSS action.

The results of the present study suggested that the combination of EPO with MPSS may have a therapeutic effect on traumatic SCI. EPO and MPSS alone and in combination significantly upregulated the mRNA and protein expression of AQP4 in astrocytes, which was decreased following oxygen and glucose deprivation. In addition, MPSS alone and in combination with EPO enhanced the viability of astrocytes following IR. The effects of EPO and MPSS on AQP4 protein expression were mitigated by blocking PKC signaling, thus suggesting that EPO and MPSS may modulate the expression of AQP4 through PKC-dependent pathways.

The concentration of EPO in the brain is closely related to hypoxia (45). In the present study, western blot analysis demonstrated that treatment with EPO or MPSS alone or in combination following ischemia, resulted in significantly enhanced AQP4 protein expression $12 \mathrm{~h}$ following reperfusion. These results suggested that EPO and MPSS may modulate water transport across the cell membrane, through the regulation of AQP4 expression, and thus prevent the onset of edema. Furthermore, EPO in combination with MPSS was revealed to protect cortical astrocytes against IR injury, as suggested by the results of the MTT assay, which indicated an increase in cell viability.

The present study demonstrated that routine recommended clinical doses had a clear effect on protein expression of AQP4. The findings suggested that combination therapy of IR injury with EPO and MPSS may have potential as a novel approach to reduce the administered doses of MPSS, and thus prevent the appearance of adverse effects. However, further studies are required to investigate the efficacy and safety of the EPO + MPSS combination therapy in eliminating edema following SCI.

\section{Acknowledgements}

The present study was supported by the Medical Science and Technology Development Foundation of the Jiangsu Province Department of Health (grant no. H200718), and the Suzhou City Social Development Fund (grant no. SSY0625).

\section{References}

1. Foreman B: The pathophysiology of delayed cerebral ischemia. J Clin Neurophysiol 33: 174-182, 2016.

2. Perez-Alvarez A, Navarrete M, Covelo A, Martin ED and Araque A: Structural and functional plasticity of astrocyte processes and dendritic spine interactions. J Neurosci 34: 12738-12744, 2014.
3. Heuser K, Szokol K and Taubøll E: The role of glial cells in epilepsy. Tidsskr Nor Laegeforen 134: 37-41, 2014 (In English, Norwegian).

4. Nesic O, Guest JD, Zivadinovic D, Narayana PA, Herrera JJ, Grill RJ, Mokkapati VU, Gelman BB and Lee J: Aquaporins in spinal cord injury: The janus face of aquaporin 4. Neuroscience 168: 1019-1035, 2010.

5. Stokum JA, Kurland DB, Gerzanich V and Simard JM: Mechanisms of astrocyte-mediated cerebral edema. Neurochem Res 40: 317-328, 2015.

6. Clasadonte J, Dong J, Hines DJ and Haydon PG: Astrocyte control of synaptic NMDA receptors contributes to the progressive development of temporal lobe epilepsy. Proc Natl Acad Sci USA 110: 17540-17545, 2013.

7. Papadopoulos MC and Verkman AS: Aquaporin water channels in the nervous system. Nat Rev Neurosci 14: 265-277, 2013.

8. Rajkowska G, Hughes J, Stockmeier CA, Javier Miguel-Hidalgo J and Maciag D: Coverage of blood vessels by astrocytic endfeet is reduced in major depressive disorder. Biol Psychiatry 73: 613-621, 2013.

9. Mao L, Wang HD, Pan H and Qiao L: Sulphoraphane enhances aquaporin-4 expression and decreases spinal cord oedema following spinal cord injury. Brain Inj 25: 300-306, 2011.

10. Wu Q, Zhang YJ, Gao JY, Li XM, Kong H, Zhang YP, Xiao M, Shields $\mathrm{CB}$ and Hu G: Aquaporin-4 mitigates retrograde degeneration of rubrospinal neurons by facilitating edema clearance and glial scar formation after spinal cord injury in mice. Mol Neurobiol 49: 1327-1337, 2014

11. Kimura A, Hsu M, Seldin M, Verkman AS, Scharfman HE and Binder DK: Protective role of aquaporin-4 water channels after contusion spinal cord injury. Ann Neurol 67: 794-801, 2010.

12. Genc S, Zadeoglulari Z, Oner MG, Genc K and Digicaylioglu M: Intranasal erythropoietin therapy in nervous system disorders. Expert Opin Drug Deliv 8: 19-32, 2011.

13. Evaniew N, Noonan VK, Fallah N, Kwon BK, Rivers CS, Ahn H, Bailey CS, Christie SD, Fourney DR, Hurlbert RJ, et al: Methylprednisolone for the treatment of patients with acute spinal cord injuries: A propensity score-matched cohort study from a Canadian multi-center spinal cord injury registry. J Neurotrauma 32: 1674-1683, 2015.

14. Wilson JR, Forgione N and Fehlings MG: Emerging therapies for acute traumatic spinal cord injury. CMAJ 185: 485-492, 2013.

15. Tesiorowski M, Potaczek T, Jasiewicz B, Sapa J and Zygmunt M: Methylprednisolone-acute spinal cord injury, benefits or risks? Postepy Hig Med Dosw (Online) 67: 601-609, 2013 (In Polish).

16. Chikuda H, Yasunaga H, Takeshita K, Horiguchi H, Kawaguchi H, Ohe K, Fushimi K and Tanaka S: Mortality and morbidity after high-dose methylprednisolone treatment in patients with acute cervical spinal cord injury: A propensity-matched analysis using a nationwide administrative database. Emerg Med J 31: 201-206, 2014.

17. Jongen PJ, Stavrakaki I, Voet B, Hoogervorst E, van Munster E, Linssen WH, Sinnige LG, Verhagen WI, Visser LH, van der Kruijk R, et al: Patient-reported adverse effects of high-dose intravenous methylprednisolone treatment: A prospective web-based multi-center study in multiple sclerosis patients with a relapse. J Neurol 263: 1641-1651, 2016.

18. Schroeder GD, Kwon BK, Eck JC, Savage JW, Hsu WK and Patel AA: Survey of cervical spine research society members on the use of high-dose steroids for acute spinal cord injuries. Spine (Phila Pa 1976) 39: 971-977, 2014.

19. Schäfer R, Mueller L, Buecheler R, Proksch B, Schwab M, Gleiter $\mathrm{CH}$ and Danielyan L: Interplay between endothelin and erythropoietin in astroglia: The role in protection against hypoxia. Int J Mol Sci 15: 2858-2875, 2014.

20. Merelli A, Czornyj L and Lazarowski A: Erythropoietin: A neuroprotective agent in cerebral hypoxia, neurodegeneration, and epilepsy. Curr Pharm Des 19: 6791-6801, 2013.

21. Ugurluer G, Cebi A, Mert H, Mert N, Serin M and Erkal HS: Neuroprotective effects of erythropoietin against oxidant injury following brain irradiation: An experimental study. Arch Med Sci 12: 1348-1353, 2016.

22. Genc S, Koroglu TF and Genc K: Erythropoietin and the nervous system. Brain Res 1000: 19-31, 2004.

23. Yuen CM, Leu S, Lee FY, Yen CH, Lin YC, Chua S, Chung SY, Chai HT, Sheu JJ, Ko SF, et al: Erythropoietin markedly attenuates brain infarct size and improves neurological function in the rat. J Investig Med 58: 893-904, 2010. 
24. Gorio A, Madaschi L, Di Stefano B, Carelli S, Di Giulio AM, De Biasi S, Coleman T, Cerami A and Brines M: Methylprednisolone neutralizes the beneficial effects of erythropoietin in experimental spinal cord injury. Proc Natl Acad Sci USA 102: 16379-16384, 2005.

25. Alibai E, Zand F, Rahimi A and Rezaianzadeh A: Erythropoietin plus methylprednisolone or methylprednisolone in the treatment of acute spinal cord injury: A preliminary report. Acta Med Iran 52: 275-279, 2014

26. Boussicault L, Hérard AS, Calingasan N, Petit F, Malgorn C, Merienne N, Jan C, Gaillard MC, Lerchundi R, Barros LF, et al: Impaired brain energy metabolism in the BACHD mouse model of Huntington's disease: Critical role of astrocyte-neuron interactions. J Cereb Blood Flow Metab 34: 1500-1510, 2014

27. He L, Zhang X, Wei X and Li Y: Progesterone attenuates aquaporin-4 expression in an astrocyte model of ischemia/reperfusion. Neurochem Res 39: 2251-2261, 2014.

28. Livak KJ and Schmittgen TD: Analysis of relative gene expression data using real-time quantitative PCR and the 2(-Delta Delta C(T)) method. Methods 25: 402-408, 2001

29. Fairbanks SL, Young JM, Nelson JW, Davis CM, Koerner IP and Alkayed NJ: Mechanism of the sex difference in neuronal ischemic cell death. Neuroscience 219: 183-191, 2012.

30. Dong W, Xiao S, Cheng M, Ye X and Zheng G: Minocycline induces protective autophagy in vascular endothelial cells exposed to an model of ischemia/reperfusion-induced injury. Biomed Rep 4: 173-177, 2016.

31. Rao KV, Reddy PV, Curtis KM and Norenberg MD: Aquaporin-4 expression in cultured astrocytes after fluid percussion injury. J Neurotrauma 28: 371-381, 2011.

32. Robertson CS, Cherian L, Shah M, Garcia R, Navarro JC, Grill RJ, Hand CC, Tian TS and Hannay HJ: Neuroprotection with an erythropoietin mimetic peptide (pHBSP) in a model of mild traumatic brain injury complicated by hemorrhagic shock. J Neurotrauma 29: 1156-1166, 2012.

33. Brissaud O, Villega F, Pieter Konsman J, Sanchez S, Raffard G, Franconi JM, Chateil JF and Bouzier-Sore AK: Short-term effect of erythropoietin on brain lesions and aquaporin-4 expression in a hypoxic-ischemic neonatal rat model assessed by magnetic resonance diffusion weighted imaging and immunohistochemistry. Pediatr Res 68: 123-127, 2010.

34. Tang Z, Sun X, Huo G, Xie Y, Shi Q, Chen S, Wang X and Liao Z: Protective effects of erythropoietin on astrocytic swelling after oxygen-glucose deprivation and reoxygenation: Mediation through AQP4 expression and MAPK pathway. Neuropharmacology 67: 8-15, 2013.
35. Miljus N, Heibeck S, Jarrar M, Micke M, Ostrowski D, Ehrenreich $\mathrm{H}$ and Heinrich R: Erythropoietin-mediated protection of insect brain neurons involves JAK and STAT but not PI3K transduction pathways. Neuroscience 258: 218-227, 2014.

36. Kwon MS, Kim MH, Kim SH, Park KD, Yoo SH, Oh IU, Pak S and Seo YJ: Erythropoietin exerts cell protective effect by activating PI3K/Akt and MAPK pathways in C6 cells. Neurol Res 36: 215-223, 2014.

37. Smith PD, Puskas F, Fullerton DA, Meng X, Cho D, Cleveland JC $\mathrm{Jr}$, Weyant MJ and Reece TB: Attenuation of spinal cord ischemia and reperfusion injury by erythropoietin. J Thorac Cardiovasc Surg 141: 256-260, 2011.

38. Hwang J, Huh J, Kim J, Jeon Y, Cho S and Han S: Pretreatment with erythropoietin attenuates the neurological injury after spinal cord ischemia. Spinal Cord 50: 208-212, 2012.

39. Chu H, Ding H, Tang Y and Dong Q: Erythropoietin protects against hemorrhagic blood-brain barrier disruption through the effects of aquaporin-4. Lab Invest 94: 1042-1053, 2014.

40. Uzüm G, Sarper Diler A, Bahcekapili N and Ziya Ziylan Y: Erythropoietin prevents the increase in blood-brain barrier permeability during pentylentetrazol induced seizures. Life Sci 78: 2571-2576, 2006 .

41. Martinez-Estrada OM, Rodriguez-Millán E, González-De Vicente E, Reina M, Vilaró S and Fabre M: Erythropoietin protects the in vitro blood-brain barrier against VEGF-induced permeability. Eur J Neurosci 18: 2538-2544, 2003.

42. Zhang C, Chen J and Lu H: Expression of aquaporin-4 and pathological characteristics of brain injury in a rat model of traumatic brain injury. Mol Med Rep 12, 7351-7357, 2015.

43. Tang G and Yang GY: Aquaporin-4: A potential therapeutic target for cerebral edema. Int J Mol Sci 17: pii:E1413, 2016.

44. Corsini E, Pinto A, Galbiati V, Viviani B, Galli CL, Marinovich M and Racchi M: Corticosteroids modulate the expression of the PKC-anchoring protein RACK-1 and cytokine release in THP-1 cells. Pharmacol Res 81: 10-16, 2014.

45. Zhang CY, Zhang JX, Lü XT and Li BY: Effects of intermittent hypoxic exposure on the parameter of erythrocyte and serum hypoxia inducible factor-1 alpha and erythropoietin levels. Xi Bao Yu Fen Zi Mian Yi Xue Za Zhi 25: 932-934, 2009 (In Chinese). 